\title{
PKS 0483-436 - A high-redshift quasar with strong X-ray absorption
}

\section{Citation}

Wilkes, Belinda J., Martin Elvis, Fabrizio Fiore, Jonathan C. McDowell, Harvey Tananbaum, and Andrew Lawrence. 1992. "PKS 0483-436 - A High-Redshift Quasar with Strong X-Ray Absorption." The Astrophysical Journal 393 (July): L1. doi:10.1086/186436.

\section{Published Version}

doi:10.1086/186436

\section{Permanent link}

http://nrs.harvard.edu/urn-3:HUL.InstRepos:30212194

\section{Terms of Use}

This article was downloaded from Harvard University's DASH repository, and is made available under the terms and conditions applicable to Other Posted Material, as set forth at http:// nrs.harvard.edu/urn-3:HUL.InstRepos:dash.current.terms-of-use\#LAA

\section{Share Your Story}

The Harvard community has made this article openly available.

Please share how this access benefits you. Submit a story.

\section{Accessibility}


The AsTrophysiCAL JournaL, 393:L1-L4, 1992 July 1

(C) 1992. The American Astronomical Society. All rights reserved. Printed in U.S.A.

\title{
PKS 0483-436: A HIGH-REDSHIFT QUASAR WITH STRONG X-RAY ABSORPTION

\author{
Belinda J. Wilkes, ${ }^{1}$ Martin Elvis, ${ }^{1}$ Fabrizio Fiore, ${ }^{1}$ Jonathan C. McDowell, ${ }^{1}$ \\ Harvey Tananbaum, ${ }^{1}$ and ANDrew LaWrence ${ }^{2}$ \\ Received 1992 January 10; accepted 1992 April 14
}

\begin{abstract}
We report the first X-ray spectrum of a high-redshift $(z=2.85)$ quasar. The ROSAT PSPC spectrum of PKS 0438-436, covering 0.3-9 keV in the quasar's rest frame, reveals unexpected absorption of $\sim 1 \times 10^{22}$ $\mathrm{cm}^{-2}$ assuming it occurs at the source. Only one other high-luminosity quasar (of $\gtrsim 50$ observed by Einstein) shows significant absorption in its X-ray spectrum. Of the common line-of-sight absorbers, only highly ionized Ly $\alpha$ forest clouds may be able to explain this amount of absorption. Candidates for an intrinsic absorber are discussed. Absorption at $\sim 1 \mathrm{keV}$ (rest frame) is due primarily to heavy elements $(\mathrm{O}, \mathrm{Ne}, \mathrm{Mg}, \mathrm{Si}, \mathrm{S})$ raising the possibility of measuring early universe abundances via X-ray absorption in this and like quasars. PKS 0438 - 436 may be a high-redshift member of a population of quasars which can contribute to the X-ray background above $2 \mathrm{keV}$, without being detectable by previous imaging missions.
\end{abstract}

Subject heading: quasars: individual (PKS 0438-436)

\section{INTRODUCTION}

$\mathrm{X}$-ray spectral observations of quasars have been confined to low-redshift objects $(z \leq 0.5)$ whose proximity makes them bright enough to study. ROSAT, with its high sensitivity, enables us to observe the spectra of high-redshift $(z>2)$ quasars for the first time. The first quasar observed in our program has given an unexpected result in that, unlike lowredshift quasars, it has significant absorption in its X-ray spectrum.

Only two quasars of the $\gtrsim 50$ studied with Einstein (Wilkes \& Elvis 1987; Canizares \& White 1989; Worrall \& Wilkes 1989) are known to have strong, soft X-ray absorption, variable in both cases: MR 2251-178 (Halpern 1984; Pan, Stewart, \& Pounds 1990), with an X-ray luminosity of $\sim 4 \times 10^{44} \mathrm{ergs}^{-1}(0.5-4.5 \mathrm{keV}$ rest frame; Wilkes et al. 1992), and NRAO 140 (Marscher 1988; Turner et al. 1991), X-ray luminosity $\sim 1 \times 10^{46} \mathrm{ergs}^{-1}(2-8 \mathrm{keV})$. In all other cases there is no detectable absorption in excess of the Galaxy, and frequently the presence of a soft excess results in a fitted $N_{\mathrm{H}}$ less than the Galactic value when a single power-law spectral form is assumed (Wilkes \& Elvis 1987). The possible presence of a soft excess adds to the uncertainty in the column density but values above a few times $10^{20} \mathrm{~cm}^{-2}$ are unlikely since they would imply an extremely strong soft excess. Significant absorption $\left(\sim 10^{22} \mathrm{~cm}^{-2}\right)$ has been seen in low-luminosity active galaxies $\left(\lesssim 10^{43}\right.$ ergs $\left.^{-1}\right)$, and it has been suggested (Lawrence \& Elvis 1982; Reichert et al. 1985; Kruper, Urry, \& Canizares 1990) that the amount of intrinsic absorption in active galaxies is inversely related to luminosity, an obvious interpretation being that the higher luminosity central source ionizes and clears out a larger portion of the host galaxy.

\section{OBSERVATIONS AND ANALYSIS}

PKS 0438-436 was selected as a high-redshift $R O S A T$ target based on its high Einstein flux compared with other similar redshift quasars in the Einstein data bank (Wilkes et al.

\footnotetext{
${ }^{1}$ Harvard-Smithsonian Center for Astrophysics, 60 Garden Street, Cambridge, MA 02138.

${ }^{2}$ Department of Physics, Queen Mary and Westfield College, Mile End Road, London E1 4NS, England, UK.
}

1992). It is a radio-loud, core-dominated, highly polarized (Impey \& Tapia 1988) quasar (Table 1). The ROSAT/PSPC observation was made with the source at the field center and the spacecraft wobble turned off (not by design) resulting in a poorly determined flux due to the unknown proportion of the observation for which the source lay under a window support wire. The source spectrum, the subject of this paper, is not affected. Counts were extracted from a 1.5 radius circle centered on the source centroid, with background estimated within an annulus of inner and outer radii $2^{\prime}$ and $4^{\prime}$, respectively. The full-width at zero intensity of the source is observed to be 1.5 and thus a circle with this radius contains $\gtrsim 95 \%$ of the counts, those lost being due to mirror scattering over the whole field. The 34 energy channels used by the MPE SASS pipeline processing were used in this analysis.

In order to provide a simple characterization of the source spectrum, a single power law with free, local $N_{\mathrm{H}}$ was fitted to the observed spectrum yielding $\alpha_{E}=0.8_{-0.3}^{+1.5}, N_{\mathrm{H}}=7.8_{-2.6}^{+26.1}$ $\times 10^{20} \mathrm{~cm}^{-2}$ with a reduced $\chi^{2}=0.88(1 \sigma$ errors, two interesting parameters [pars], channels $5: 34^{3}$ ). The latest version of the PSPC response matrix was used for this fit, i.e., that released in 1992 February and recently installed in the SASS processing system (Snowden et al. 1992). The level of uncertainty in this matrix is $\$ 4 \%$ based on fits to the Crab and HZ 43 calibration data (S. L. Snowden, private communication), which is insignificant when compared with $\sim 20 \%$ statistical errors per channel in the current spectrum. The bestfit spectrum is shown in Figure $1 a$ with the data superposed and the residuals beneath. Figure $1 b$ shows the $68 \%, 90 \%$, and $99 \% \alpha_{E}, N_{\mathrm{H}}$ confidence contours for the fit. We note that, while the data do not require a spectrum more complex than this simple power law, it is not a unique fit to the data but rather a convenient and simple representation.

As noted above, due to the lack of spacecraft wobble for this observation, estimates of the source flux are unreliable as the fraction of the observation for which the source may have been occulted by one of the window support wires is uncertain. The count rate for our target is low $\left(\sim 0.06 \mathrm{~s}^{-1}\right)$ so that its light curve shows no clear indication of such occultation. The flux

\footnotetext{
${ }^{3}$ Channels $1: 4$ contain no significant signal.
} 

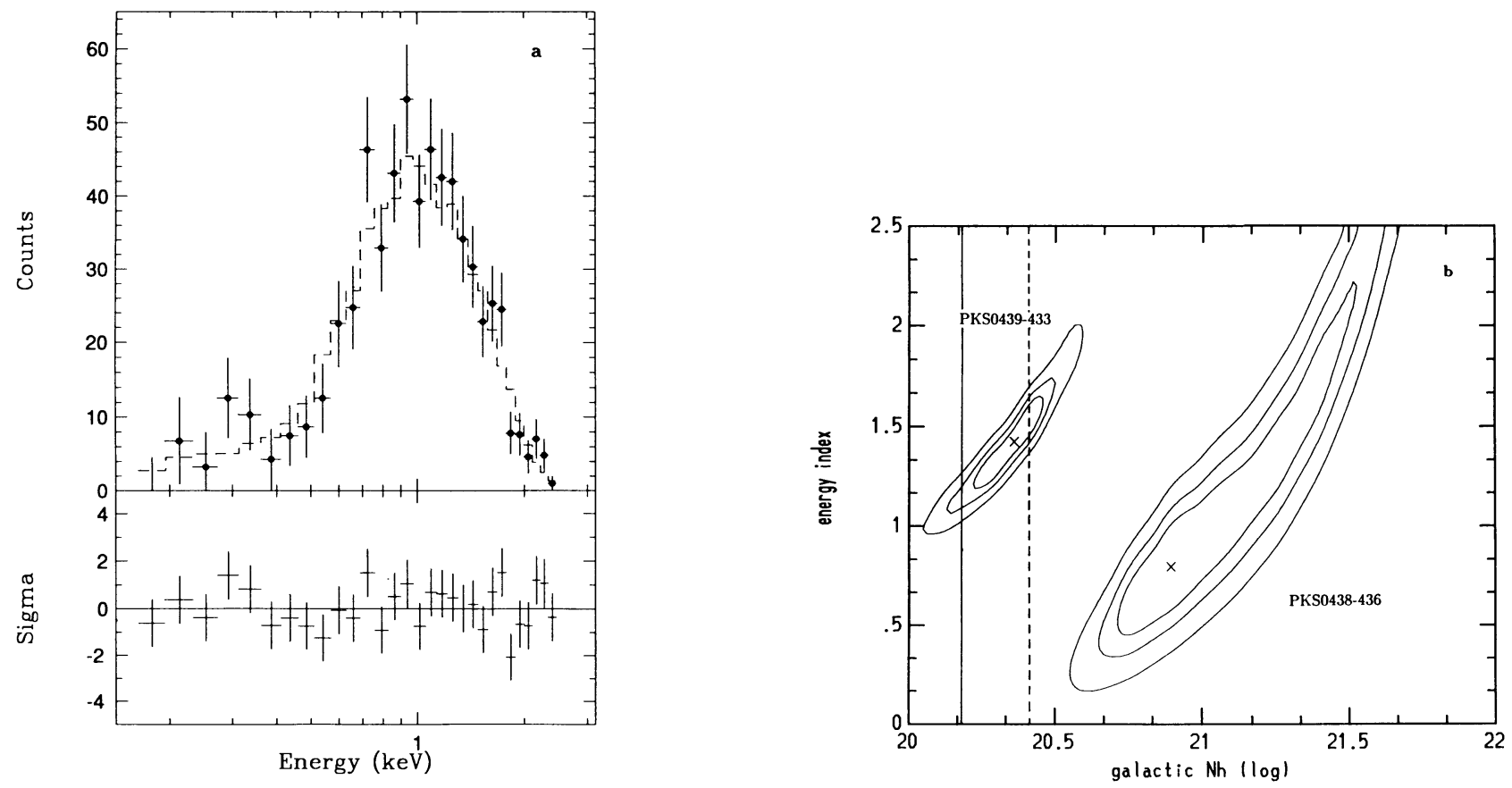

FIG. 1.- (a) The best fit for a single power-law spectral fit with free local $N_{\mathrm{H}}$ to the spectrum of PKS $0438-436$. (b) The $68 \%$, $90 \%$ and $99 \%$ confidence contours for the same fit for both PKS $0438-436$, PKS 0439-433. Galactic $N_{\mathrm{H}}, N_{\mathrm{H}}+1 \sigma$ are indicated by vertical lines.

indicated by the spectral fits is $1.5 \times 10^{-12}$ ergs $\mathrm{cm}^{-2} \mathrm{~s}^{-1}$ $(0.1-2.5 \mathrm{keV})$ but could be as high as twice this value.

The equivalent neutral hydrogen absorbing column density, determined under the assumption of solar abundances based on the amount of metal absorption observed in the X-ray spectrum, is significantly larger than the measured Galactic column (Fig. 1b). A second quasar in the same PSPC field, PKS 0439-433 (Table 1), shows a spectrum, with similar signal-tonoise ratio $(\mathrm{S} / \mathrm{N})$, consistent with the Galactic column density (Fig. $1 b, \alpha_{E}=1.4 \pm 0.3, N_{\mathbf{H}}=2.3_{-0.7}^{+0.8}, \chi^{2}=0.73$, channels $1: 34,1 \sigma, 2$ pars.). ${ }^{4}$ The spectrum of the subtracted background is similar for both quasars. This result precludes the presence of an additional local absorber or of systematic effects in the data itself. ${ }^{5}$ We conclude that the excess absorption in the spectrum of PKS 0438-436 must lie either outside our Galaxy along the line of sight, or be intrinsic to the quasar. The amount of material implied depends on the redshift at which it is situated. If it is intrinsic, the rest frame energy seen by $R O S A T$ is $0.3-9 \mathrm{keV}$ with the spectrum absorbed up to $2 \mathrm{keV}$. A spectral fit with local absorption fixed at the measured Galactic value plus free absorption at $z=2.85$ yields no

${ }^{4}$ We note that the PKS 0439-433 data show no indication of the presence of a soft excess, and no good fit could be found with a broken power-law model, $\chi_{\min }^{2} \sim 50$.

5 The light curves of PKS 0438-436 show no systematic effects which could lead to the observed absorption. change in slope $\left(\alpha_{E}=0.7_{-0.3}^{+0.7}\right)$ and an intrinsic absorption column of $1.0_{-0.4}^{+0.7} \times 10^{22} \mathrm{~cm}^{-2}$ (reduced $\chi^{2}=0.83$, Fig. $2 a$ ). We then made a series of spectral fits with two absorbing components, one fixed at the Galactic column density with redshift zero, ${ }^{6}$ and the second free to vary and placed at a range of redshifts up to 2.85 . The resulting dependence of the deduced column density of the absorber upon its redshift is shown in Figure $2 b$.

\section{RESULTS}

PKS 0438-436 with an X-ray luminosity in the range $1.7 \pm 0.1 \times 10^{47}$ ergs $^{-1}$ (Einstein, $0.5-4.5 \mathrm{keV}$; Wilkes et al. $1992) \sim 3-6 \times 10^{47}$ ergs $^{-1}(R O S A T)$ is the highest luminosity quasar with significant soft $\mathrm{X}$-ray absorption. Along with NRAO 140, it does not follow the previously reported inverse correlation between luminosity and X-ray absorption unless the absorption should prove to be along the line of sight rather than intrinsic to the quasar.

PKS $0438-436$ was also observed by the Einstein Observatory on 1979 August 26. $160 \pm 18$ counts were detected and, with a flux of $9.0 \pm 0.1 \times 10^{-\overline{13}} \mathrm{ergs} \mathrm{cm}^{-2} \mathrm{~s}^{-1}(0.16-3.5 \mathrm{keV}$; Wilkes et al. 1992), was one of the brightest, high-redshift quasars observed. This flux level is roughly half that seen by

${ }^{6}$ Allowing the local absorber to be free results in no useful constraints on the spectral parameters. The best-fit values do not change significantly.

TABLE 1

Quasar Properties

\begin{tabular}{|c|c|c|c|c|c|c|c|c|}
\hline \multirow[b]{2}{*}{ NAME } & \multirow[b]{2}{*}{ R.A. (J2000) } & \multirow[b]{2}{*}{ DECL. (J2000) } & \multirow[b]{2}{*}{$\boldsymbol{V}$} & \multirow[b]{2}{*}{$z$} & \multirow[b]{2}{*}{$\begin{array}{c}N_{\mathrm{H}}(\mathrm{Gal})^{\mathrm{a}} \\
\left(\mathrm{cm}^{-2}\right)\end{array}$} & \multicolumn{2}{|c|}{$R O S A T$} & \multirow[b]{2}{*}{$\begin{array}{c}\text { Net } \\
\text { Counts }\end{array}$} \\
\hline & & & & & & Date & Time (s) & \\
\hline PKS $0438-436 \ldots \ldots$ & $04^{\mathrm{h}} 40^{\mathrm{m}} 17^{\mathrm{s}} .18$ & $-43^{\circ} 33^{\prime} 09^{\prime \prime} 0$ & 18.8 & 2.85 & $1.5 \pm 1.0$ & 1991 Feb 19-21 & 10725 & $625 \pm 28$ \\
\hline PKS $0439-433 \ldots \ldots$ & $0441 \quad 16.1$ & -431328 & 16.4 & 0.593 & $1.5 \pm 1.0$ & 1991 Feb $19-21$ & 10725 & $1028 \pm 41$ \\
\hline
\end{tabular}

${ }^{a}$ Heiles \& Cleary 1979. 

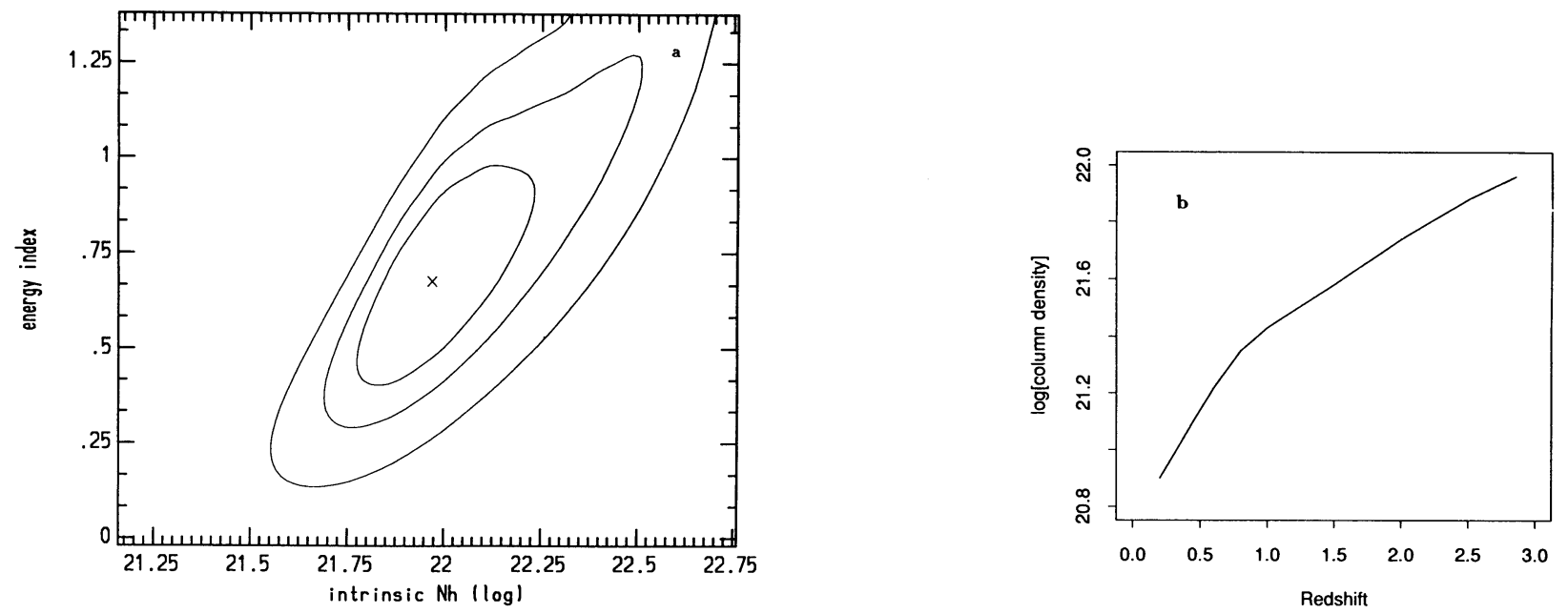

FIG. 2.- (a) The confidence contours for a single power-law fit assuming Galactic plus intrinsic $N_{\mathrm{H}}$ for PKS 0438-436. (b) The dependence of the deduced equivalent hydrogen column density on the redshift assumed for the absorber.

ROSAT suggesting variation as the ROSAT value is probably a lower limit. The $\mathrm{S} / \mathrm{N}$ of the observation is too poor to obtain a constraint on the spectral parameters beyond local $N_{\mathrm{H}} \lesssim$ $2 \times 10^{22} \mathrm{~cm}^{-2}$, although the best-fit value of $N_{\mathrm{H}} \sim 8 \times 10^{20}$ $\mathrm{cm}^{-2}$ is comparable with the value from ROSAT (Canizares \& White 1989).

The X-ray energy spectral index of PKS $0438-433\left(\alpha_{E} \sim\right.$ $0.3)$ is consistent with those observed for radio-loud and highly polarized quasars (Wilkes \& Elvis 1987; Worrall \& Wilkes 1989). However, it is flatter than the typical 1.2 energy index recorded, mostly for radio-quiet quasars, by ROSAT (Brinkman et al. 1992). There is some evidence from ROSAT in both survey and deep pointed data that the quasar spectral slopes flatten toward higher redshift (Brinkman et al. 1992; Shanks et al. 1991).

The presence of a strong absorber is not a unique interpretation of these observations. Instead, the spectrum may be complex, for example with spectral flattening toward lower energies, as observed in BL Lac objects (Madejski et al. 1992). A broken power-law fit to PKS 0438-436 gives a break energy of $0.9 \pm 0.2 \mathrm{keV}$ and a low-energy slope $\alpha_{E}=1.2 \pm 0.6$ $\left(\chi^{2}=0.81\right.$, no significant improvement over a single power-law model). The extreme change in slope ( $\Delta \alpha \sim 2$, compared to 0.7 for BL Lac objects) and the higher break energy (3.0, compared to $1.5 \mathrm{keV}$, rest frame), along with the lack of any similar observations for other flat-spectrum, radio-loud quasars make this an unlikely interpretation.

\section{IDENTITY OF THE ABSORBER}

The ROSAT/PSPC spectral resolution does not allow us to distinguish between excess absorption along the line of sight or intrinsic to the quasar so both possibilities are considered.

\subsection{Line-of-Sight Absorption}

Absorption along the line of sight to quasars is a well-known and heavily studied phenomenon with "Ly $\alpha$ forest " and metalline systems being the dominant sources. The former typically have low neutral hydrogen column densities $\left(\sim 10^{14} \mathrm{~cm}^{-2}\right)$ which, even integrated over $\Delta z \sim 3$, cannot explain that observed in the soft X-ray. Their uncertain level of ionization, with estimates of the neutral to ionized gas ratio as low as $10^{-5}$ to $10^{-6}$ (Chokshi 1992), narrows the discrepancy but the lack of metal absorption lines is inconsistent with the strong metal absorption in the X-ray. The higher column density $\left(\sim 10^{18} \mathrm{~cm}^{-2}\right)$ metal-line systems, with ionization $\sim 10^{-3}$, are a stronger possibility but their much lower density, $\sim 1.2$ per redshift interval (Sargent, Steidel, \& Boksenberg 1989), again falls short of the X-ray measurements.

Higher column density absorbers which are also fairly common are the Ly $\alpha$ disk systems (characterized by damped Ly $\alpha$ absorption lines; see Turnshek et al. 1989 and references therein). The optical spectrum of PKS $0438-436$ shows no strong $\operatorname{Ly} \alpha$ absorption down to $3500 \AA$ (i.e., $z=1.88$; Morton, Savage, \& Bolton 1978) so that any such system must be situated at $z<1.88$. Searches for Ly $\alpha$ disk absorption have indicated that such systems, typically with column densities $\sim 10^{20}-10^{21} \mathrm{~cm}^{-2}$, occur at the rate of $d N / d z=0.29 \pm 0.07$ (Turnshek et al. 1989). Thus, if we assume that there is no evolution in Ly $\alpha$ disk systems, there is a $55 \%$ chance of one existing at $z<1.88$ along the line of sight to PKS $0438-436$. Given that a single system would provide insufficient column density unless it is at $z<0.4$ (Fig. $2 b$ ) and that all systems found to date are at $z \sim 2$, it seems unlikely that Ly $\alpha$ disks are responsible for the absorption we see.

Another possibility for absorption along the line of sight is the presence of dust in intervening galaxies which has been suggested to account for the lack of high-redshift quasars in current surveys (Heisler \& Ostriker 1988). However, the prediction is that, for quasars brighter than $22 \mathrm{mag}$, the reddening is minimal. Specifically, at $z=2.85$, the predicted mean absorption has $E_{B-V} \sim 0.15 \pm 0.13$, while the $N_{\mathrm{H}}$ we observe indicates $E_{B-V}$ of $\sim 1, \mathrm{a} \sim 6 \sigma$ deviation above this. The presence of dust in intervening galaxies is unlikely to explain the large column is PKS 0438-436.

\subsection{Intrinsic Absorption}

The alternative of intrinsic absorption may be related to its high redshift, high luminosity, or the apparent importance of beaming. Little previous X-ray spectral information exists for any of these regimes. Typical intrinsic X-ray column densities seen in low luminosity active galaxies are also $\sim 10^{22} \mathrm{~cm}^{-2}$ so that the detection of a similar column in a high-luminosity object may not be surprising. If PKS $0438-436$ were at low redshift the absorption would have reduced its soft X-ray flux by a factor $\sim 10$, so that an object with comparable bolometric flux would not have been detected by Einstein or ROSAT. The 
lack of observations of such systems to date could be a selection effect due to the higher X-ray flux limit of previous missions.

PKS 0438 - 436 has no broad absorption lines, ruling out a broad absorption-line cloud as an absorption candidate. It does have broad emission lines, so a cloud in this region, which would have roughly the correct column density $\left(2.5 \times 10^{22}\right.$ $\mathrm{cm}^{-2}$; Kwan \& Krolik 1981) is a possibility. However, their typical size, $\sim 10^{13} \mathrm{~cm}$, is likely smaller than the X-ray source implying partial covering. Leakage of soft photons would then be expected whereas the X-ray spectrum shows no evidence for this, a covering factor of more than 0.8 is required. The absorbing cloud must therefore be larger than the typical broad-line region cloud.

Both PKS 0438-436 and NRAO 140 have very strong, core-dominated radio emission and high optical polarization, which implies relativistic beaming toward us. Naïvely, one would expect such objects to be less likely to contain intrinsic absorbing material along our line of sight. However, the BL Lac object PKS 2155-304, which has many similar properties, also has unexpected X-ray absorption (Canizares \& Kruper 1984), as do other BL Lac objects (Madejski et al. 1991, 1992). This absorption has been identified as an O vIII Ly $\alpha$ absorption feature at low redshift $(z<0.15$ for PKS $2155-304)$ from hot $\left(\sim 10^{6} \mathrm{~K}\right)$ or highly ionized material with a column density of $2 \times 10^{22} \mathrm{~cm}^{-2}$. The material is likely to be situated in or close to the beam of the radio source and is required to be in this very hot or very high ionized state due to the presence of only a single absorption line. The absorption in PKS $0438-436$ is broad and show no evidence for recovery at low energies, implying cooler material $\left(\lesssim 10^{6} \mathrm{~K}\right.$ for coronal ionization and $\lesssim 10^{4} \mathrm{~K}$ for photoionization equilibrium; Kallman \& Krolik 1986). Alternatively, as suggested by the referee, the material could be highly ionized and have very high velocities $(\sim 0.8 c)$ generating a broad absorption-line analogous to those in BAL quasars. The absorption in PKS 2155-304 has a width of $\sim 0.1 c$.

If the intrinsic absorption is evolutionary (i.e., due to its high redshift), PKS 0438-436 may be a high-redshift analog of the ultraluminous infrared galaxies discovered by IRAS (Sanders et al. 1988). These are suggested to be young quasars in a dust-enshrouded initial phase. The $E_{B-V}$ values deduced from the emission-line ratios for the IRAS galaxies are in the range $0.6-2.3$, in good agreement with the X-ray column density of PKS 0438-436.

With only one high-redshift quasar observed, it is impossible to distinguish between these various candidate absorbers.
Clearly, X-ray spectra of more objects are required to further study this phenomenon.

\section{DISCUSSION}

It seems likely that the strong, soft X-ray absorption is intrinsic to PKS 0438 - 436. Whether intrinsic/extrinsic, its discovery at high redshifts provides us with a new opportunity to study the metallicity of gas since X-ray absorption is strongly metal sensitive. Optical lines in emission or absorption, the only metallicity indicator to date, have proved unreliable due to uncertainties in the ionization conditions and to saturation and blending of the lines. The X-ray absorption column is, however, a direct measure of the K-edge strengths of $\mathrm{O}, \mathrm{Ne}$, $\mathrm{Mg}, \mathrm{Si}, \mathrm{S}$, and $\mathrm{Fe}$ and is insensitive to ionization state as long as the gas is not fully ionized. With higher resolution spectra the edges of the individual elements can be measured. From the current observation alone we cannot place limits on the evolution of the metallicity with redshift since we have no independent measure of the hydrogen column density, although we can conclude, unsurprisingly given quasar emission lines, that metals exist at redshift $\sim 3$. If the metallicity were significantly reduced below local values, then the equivalent hydrogen column density deduced from this observation would be even higher than that discussed here. It may be possible to place a weak lower limit on the metallicity since, if it were reduced to $\lesssim 1 \%$ of solar value, the deduced column density would $\gtrsim 10^{24} \mathrm{~cm}^{-2}$ and Compton scattering would become important (Kallman \& Mushotzky 1985). As we observe more highredshift quasars, a study of the distribution of intrinsic $N_{\mathrm{H}}$ values at a range of redshifts will give us information on the evolution of the metallicity with redshift.

The existence of an obscured population of quasars with column densities of $\sim 10^{22} \mathrm{~cm}^{-2}$ has also been suggested to reconcile the Einstein Extended Medium Sensitivity Survey number counts (Gioia et al. 1990) with those from Ginga fluctuation analysis at higher energies (Warwick \& Stewart 1990). Based on our results for PKS 0438-436, this obscured population may encompass the whole AGN population rather than only low-luminosity objects as originally suggested.

This work was carried out with the financial support of grants: NAG 5-1724, NAG 5-1536, NAGW2201, and has made use of the NASA/IPAC Extragalactic Data base (NED) which is operated by the Jet Propulsion Laboratory, California Institute of Technology, under contract with the National Aeronautics and Space Administration.

\section{REFERENCES}

Brinkmann, W., et al. 1992, in Proc. MPE Conf., AGN and the X-Ray Background, in press

Canizares, C. R., \& Kruper, J. 1984, ApJ, 278, L99

Canizares, C. R., \& White, J. L. 1989, ApJ, 339, 27

Chokshi, A. 1992, ApJ, submitted

Gioia, I. M., Maccacaro, T., Schild, R. E., Wolter, A., Stocke, J. T., Morris, S. L., \& Henry, J. P. 1990, ApJS, 72, 567

Halpern, J. P. 1984, ApJ, 281, 90

Heiles, C., \& Cleary, M. N. 1979, Australian J. Phys. Suppl., 47, 1

Heisler, J., \& Ostriker, J. P. 1988, ApJ, 332, 543

Impey, C. D., \& Tapia, S. 1988, ApJ, 333, 666

Kallman, T., \& Krolik, J. 1986, ApJ, 308, 805

Kallman, T. R., \& Mushotzky, R.F. 1985, ApJ, 292, 49

Kruper, J. S. Urry, C. M. \& Canizares, C. R. 1990, ApJS, 74, 347

Kwan, J., \& Krolik, J. H. 1981, ApJ, 250, 478

Lawrence, A., \& Elvis, M. 1982, ApJ, 256, 410

Madejski, G., et al. 1992, in Proc. Frontiers of X-ray Astronomy, ed. K. Koyama (Universal Academic), in press

Madejski, G. M., Mushotzky, R. F., Weaver, K. A., Arnaud, K. A., \& Urry, C. M. 1991, ApJ, 370, 198
Marscher, A. P. 1988, ApJ, 334, 552

Morton, D. C., Savage, A., \& Bolton, J. G. 1978, MNRAS, 185, 735

Reichert, G. A., Mushotzky, R. F., Petre, R., \& Holt, S. S. 1985, ApJ, 296, 69

Pan, H. C., Stewart, G. C., \& Pounds, K. A. 1990, MNRAS, 242, 177

Sanders, D. B., Soifer, T. B., Elias, J. H., Madore, B. F., Matthews, K., Neugebauer, G., \& Scoville, N. Z. 1988, ApJ, 325, 74

Sargent, W. L. W., Steidel, C. C., \& Boksenberg, A. 1989, ApJS, 69, 703

Shanks, T., Georgantopoulos, I., Stewart, G. C., Pounds, K. A., Boyle, B. J., \& Griffiths, R. E. 1991, Nature, 353, 315

Snowden, S. L., et al. 1992, in preparation

Turner, T. J., Weaver, K. A., Mushotzky, R. F., Holt, S. S., \& Madejski, G. M. 1991, ApJ, 381, 85

Turnshek, D. A., Wolfe, A. M., Lanzetta, K. M., Briggs, F. H., Cohen, R. D., Foltz, C. B., Smith, H. E., \& Wilkes, B. J. 1989, ApJ, 344, 567

Warwick, R. S., \& Stewart, G. S. 1990, in Proc. 23d ESLAB Symp., ed N. White (Noordwijk: ESA Pub. Div.), 2, 727

Wilkes, B. J., \& Elvis, M. 1987, ApJ, 323, 243

Wilkes, B. J., Tananbaum, H., Worrall, D. M., Avni, Y., Oey, M. S., \& Flanagan, J. 1992, ApJS, in preparation

Worrall, D. M., \& Wilkes, B. J. 1989, ApJ, 360, 396 